\title{
Accurate Matrix Multiplication on Binary128 Format Accelerated by Ozaki Scheme
}

\author{
Daichi Mukunoki \\ daichi.mukunoki@riken.jp \\ RIKEN Center for Computational Science \\ Kobe, Hyogo, Japan \\ Takeshi Ogita \\ ogita@lab.twcu.ac.jp \\ Tokyo Woman's Christian University \\ Suginami, Tokyo, Japan
}

\author{
Katsuhisa Ozaki \\ ozaki@sic.shibaura-it.ac.jp \\ Shibaura Institute of Technology \\ Saitama, Saitama, Japan \\ Toshiyuki Imamura \\ imamura.toshiyuki@riken.jp \\ RIKEN Center for Computational Science \\ Kobe, Hyogo, Japan
}

\begin{abstract}
Although IEEE 754-2008 binary128 (with a 15-bit exponent and 113-bit significand, i.e., quadruple-precision) is not currently implemented on x86 in hardware, software emulation is available on some compilers. However, the performance is significantly slower compared to the binary64 operation, which is supported natively in hardware. This study proposes a fast implementation of matrix multiplication on matrices stored in the binary 128 format on $\mathrm{x} 86$ CPUs. The proposed implementation utilizes the Ozaki scheme, which is an accurate matrix multiplication algorithm proposed by Ozaki et al. in 2012. This scheme enables one to perform most computations using the binary64 matrix multiplication (the DGEMM routine in Basic Linear Algebra Subprograms (BLAS)); it can exploit the high-performance of highly-optimized vendor BLAS. Although the achievable performance depends on the input matrices (the inner-product dimension, the absolute range, and the significand bit length), the proposed implementation can achieve better performance and accuracy compared to naive matrix multiplication performed using the GCC's binary128 emulation in many cases. In addition, we discuss GPU acceleration, performance on reduced precision inputs, an implementation based on binary32 matrix multiplication (SGEMM), application to memory-intensive operations, and the possibility of a distributed parallel implementation.
\end{abstract}

\section{CCS CONCEPTS}

- Computer systems organization $\rightarrow$ Embedded systems; Redundancy; Robotics; • Networks $\rightarrow$ Network reliability.

\section{KEYWORDS}

Binary128, FP128, Quadruple precision, Accurate, Matrix multiplication, Linear algebra, BLAS

\section{ACM Reference Format:}

Daichi Mukunoki, Katsuhisa Ozaki, Takeshi Ogita, and Toshiyuki Imamura. 2021. Accurate Matrix Multiplication on Binary128 Format Accelerated by

Permission to make digital or hard copies of part or all of this work for personal or classroom use is granted without fee provided that copies are not made or distributed for profit or commercial advantage and that copies bear this notice and the full citation on the first page. Copyrights for third-party components of this work must be honored

For all other uses, contact the owner/author(s).

ICPP '21, August 9-12, 2021, Lemont, IL, USA

(C) 2021 Copyright held by the owner/author(s).

ACM ISBN 978-1-4503-9068-2/21/08.

https://doi.org/10.1145/3472456.3472493
Ozaki Scheme. In 50th International Conference on Parallel Processing (ICPP '21), August 9-12, 2021, Lemont, IL, USA. ACM, New York, NY, USA, 11 pages. https://doi.org/10.1145/3472456.3472493

\section{INTRODUCTION}

Most modern numerical computations use 32-bit (single-precision) or 64-bit (double-precision) floating-point operations; however, 128bit (quadruple-precision) floating-point operations are required for some computations that need a wider range of exponent and/or significand [2][32][22]. In addition, as the hardware performance has improved, the scale of computation has increased, and there is concern that the accuracy of computation results may decrease due to the accumulation of rounding errors in large-scale problems. High-precision arithmetic is the most basic approach to this problem. In 2008, the binary128 format was introduced in IEEE 754-2008 [17] (Table 1). Currently, binary128 is not implemented in hardware on most processors, including x86, ARM, and GPUs; however, several software implementations (emulations) are available [11]. Most commonly, the GNU C compiler (GCC) supports binary128 as the _-_ float128 type ${ }^{1}$, and the GCC Quad-Precision Math Library (libquadmath) ${ }^{2}$ provides mathematical functions for binary128 (equivalent to libmath). However, as we demonstrate in this paper, the performance of software implementations of binary128 is extremely slow compared to that of binary64, and it takes an enormous amount of time to perform large computationally intensive computations.

This paper proposes a fast implementation of matrix multiplication on matrices stored in the binary 128 format on x 86 CPUs. Matrix multiplication is a fundamental and computationally intensive kernel of linear algebra known as the GEMM routine in level-3 Basic Linear Algebra Subprograms (BLAS) [9]. The proposed implementation partially uses binary128 emulation; however, by utilizing the Ozaki scheme [34], which is an error-free transformation for matrix multiplication, we can perform most computations using binary64 matrix multiplication (DGEMM). By building on vendor-optimized DGEMM, it is possible to achieve high performance and eliminate parallelization and optimization costs. We demonstrate that the proposed implementation can outperform existing binary128 and double-double GEMM implementations with a naive matrix multiplication algorithm with a triple-loop on $\mathrm{x} 86$ processors. In addition,

\footnotetext{
${ }^{1}$ https://gcc.gnu.org/onlinedocs/gcc/Floating-Types.html

${ }^{2}$ https://gcc.gnu.org/onlinedocs/libquadmath/
} 
Table 1: Binary floating-point formats in IEEE 754-2008. The significand includes the hidden 1 bit. Min and Max are the normalized numbers.

\begin{tabular}{l|rrrlll}
\hline Format & Exponent (bits) & Significand (bits) & Decimal digits & Min & Max & Unit round-off \\
\hline binary16 (FP16) & 5 & 11 & 3.31 & $6.1 \mathrm{E}-5$ & $6.6 \mathrm{E}+4$ & $4.9 \mathrm{E}-4$ \\
binary32 (FP32) & 8 & 24 & 7.22 & $1.2 \mathrm{E}-38$ & $3.4 \mathrm{E}+38$ & $6.0 \mathrm{E}-8$ \\
binary64 (FP64) & 11 & 53 & 15.95 & $2.2 \mathrm{E}-308$ & $1.8 \mathrm{E}+308$ & $1.1 \mathrm{E}-16$ \\
binary128 (FP128) & 15 & 113 & 34.02 & $3.4 \mathrm{E}-4932$ & $1.2 \mathrm{E}+4932$ & $9.6 \mathrm{E}-35$ \\
\hline
\end{tabular}

the proposed implementation achieves higher accuracy than naive matrix multiplication with the binary128 arithmetic. Furthermore, we discuss the following as potential applications and extensions of the proposed implementation.

- GPU acceleration (by offloading DGEMMs).

- Binary32 GEMM (SGEMM)-based implementation (use SGEMM instead of DGEMM for most computations).

- Reduced-precision performance (higher performance can be achieved when the input matrices are double or triple precision than when the input matrices are quadruple precision).

- Matrix-vector multiplication (as an example for memory-intensive operations).

- Distributed parallel implementation (possibilities for PxGEMM equivalent implementation (without demonstration)).

A DGEMM implementation using Tensor Core GEMM [26] (Tensor Cores are matrix multipliers that can operate binary16 input data with binary32 accumulator on NVIDIA GPUs) has been proposed as an emulation of high-precision matrix multiplication via low-precision matrix multiplication using the Ozaki scheme. Compared to this previous study, this paper provides the following contributions.

- We apply the Ozaki scheme to high-precision (binary128) computations in environments where high-precision operations are not supported on hardware.

- We present specific optimization methods to compute binary 128 using binary64.

- We present an implementation and evaluation on x86 hardware, while the previous study targeted NVIDIA GPUs.

- We discuss applications and extensions of the proposed implementation, i.e., GPU acceleration, an SGEMM-based implementation, reduced-precision performance, matrix-vector multiplication, and a distributed parallel implementation.

The remainder of this paper is organized as follows. Section 2 introduces related work. Section 3 describes the method, which is based on the Ozaki scheme. Section 4 presents optimization methods. Section 5 presents experimental results on Intel Skylake CPUs. Section 6 discusses several extensions of the proposed implementation. Finally, conclusions are presented in Section 7.

\section{RELATED WORK}

Binary128 software emulation is supported by several compilers; thus, we can easily convert code (written with binary64) to a binary128 version by simply replacing the data type. However, this can sometimes result in unintended behavior on, for example, numerical solvers. Therefore, as a validated implementation,
MPLAPACK ${ }^{3}$ [31] was developed as a multi-precision BLAS and Linear Algebra PACKage (LAPACK) [1] that officially supports binary128 emulation. It also supports other high-precision arithmetic libraries, e.g., GNU Multiple Precision Arithmetic Library $(\mathrm{GMP})^{4}$, the GNU Multiple Precision Floating-Point Reliable Library $(\mathrm{MPFR})^{5}$ [10], and $\mathrm{QD}^{6}$ [13]. MPLAPACK does not provide SIMDoptimized routines (unlike vendor BLAS); however, it is parallelized with OpenMP.

For quadruple-precision floating-point arithmetic, double-double (DD) arithmetic [6][12] is a lightweight method that is faster than binary128 emulation. However, DD arithmetic is incompatible with binary128. It represents a quadruple precision number by concatenating two binary64 values and computes the arithmetic using binary64 operations. Accordingly, its format is 106-bit significand with 11-bit exponent. Here, the significand is shorter than binary128, and the exponent is not extended. This principle can be extended by combining three, four, or more binary 64 values, e.g., the quaddouble (QD) arithmetic, which is available in the QD library, combines four binary64 values. The DD arithmetic is available on several high-precision arithmetic libraries, e.g., the QD library and CAMPARY $^{7}$ [19] (for CUDA). For linear algebra operations, in addition to MPLAPACK, QPBLAS ${ }^{8}$ and QPBLAS-GPU ${ }^{9}$ are available as BLAS implementations. XBLAS ${ }^{10}$ [21] also provides twice the precision length of binary64 based on the same principle as DD, but only supports binary64 type inputs and outputs. In addition, although not the full set of BLAS, several implementations and performance optimizations of GEMM have been reported, for example, on x86 (AMD EPYC) with AVX2 [36], on NVIDIA Fermi GPUs [27], on AMD Cypress GPUs [28], and on PEZY-SC2 many-core processors [14]. According to these reports, a properly optimized GEMM with DD arithmetic can achieve performance that is comparable to its theoretical peak performance, which is roughly 20 times the execution time of DGEMM (there are several variants of DD arithmetic and their performance depends on the accuracy obtained); however, performance optimization is not easy in SIMD architectures because SIMD-vectorization must be performed manually and is required to achieve high performance.

In addition, several BLAS implementations that can perform accurate operations to achieve numerical reproducibility have been

\footnotetext{
https://github.com/nakatamaho/mplapack

${ }^{4}$ https://gmplib.org/

${ }^{5}$ https://www.mpfr.org/

${ }^{6}$ https://www.davidhbailey.com/dhbsoftware/

${ }^{7} \mathrm{http}: / /$ homepages.laas.fr/mmjoldes/campary/

${ }^{8} \mathrm{https} / / /$ ccse.jaea.go.jp/software/QPBLAS/index_eng.html

${ }^{9} \mathrm{https}$ ://ccse.jaea.go.jp/software/QPBLAS-GPU/index_eng.html

${ }^{10}$ https://www.netlib.org/xblas/
} 
developed. For example, ExBLAS ${ }^{11}$ [15] combines accurate arithmetic similar to DD arithmetic with a long accumulator, OzBLAS ${ }^{12}$ [24] uses the same principle as in this study, and ReproBLAS ${ }^{13}$ [7] combines multiple accumulators with pre-rounding. The first two can perform infinite precision operations and support CPUs and GPUs; however, they target binary64 as the inputs and outputs. In addition, High-Precision Anchored (HPA) Accumulators [3] for accurate and reproducible computations and the Residue Number System (RNS) [18], which is an arbitrary precision operation that can be a counterpart of MPFR, have been implemented previously.

From a hardware perspective, some IBM processors, e.g., the Power9 processor, support binary128 operations; however, to the best of our knowledge, there is no trend to support binary128 on other general-purpose processors. In the Gravity Pipe (GRAPE) series, which is an accelerator series for gravitational computations, GRAPE-MP [29] supports quadruple precision floating-point operations (but is not compatible with binary128). In addition, Field Programmable Gate Arrays (FPGAs), which have been increasingly applied to high-performance computing, can implement any customized floating-point arithmetic unit, and binary128 has also been implemented [30].

Other studies related to the Ozaki scheme, which is the key method of this study, are discussed in Sections 3 and 4.

\section{METHODOLOGY}

This study utilizes the Ozaki scheme, which is an accurate matrix multiplication algorithm based on error-free transformation of inner product and matrix multiplication, proposed by Ozaki et al. in 2012 [34]. Note that we apply the modified Ozaki scheme to compute DGEMM using Tensor Core GEMM [26] to computation of binary128 GEMM using DGEMM. There are no algorithmic changes except for the difference in the precisions.

Here, we explain the scheme on the inner product; however, this scheme can be naturally extended to any inner product-based operations, including matrix-matrix multiplication. The principle of this method can be understood as an extension of high-precision arithmetic with multiple components (e.g., DD arithmetic and QD arithmetic) to the vector level. Briefly, this method comprises the following three steps.

Step.1. Element-wise splitting of the input vectors into several split vectors.

Step.2. Computation of the all-to-all products of the split vectors.

Step.3. Element-wise summation (reduction) of the above inner product results.

Here, $\mathbb{F}_{\mathrm{b} 128}$ denotes the set of binary128 floating-point numbers, $\mathrm{fl}_{\mathrm{b} 128}(\cdot)$ and $\mathrm{fl}_{\mathrm{b} 64}(\cdot)$ denote the binary128 and binary64 floatingpoint operations with the round-to-the-nearest, respectively, and $u_{b 128}$ and $u_{b 64}$ represent the unit round-offs of binary128 and binary64, respectively.

To compute the inner product of $\boldsymbol{x}, \boldsymbol{y} \in \mathbb{F}_{\mathrm{b} 128}{ }^{n}$, both input vectors are first split into several vectors in an element-wise manner.

\footnotetext{
$\overline{{ }^{11} \text { https://github.com/riakymch/exblas }}$

${ }^{12} \mathrm{http}: / /$ www.math.twcu.ac.jp/ogita/post-k/results.html

${ }^{13}$ https://bebop.cs.berkeley.edu/reproblas/
}

For $\boldsymbol{x}$ with constant $\rho$ defined in Equation (1), we perform Equations (2)-(6) from $p=1$ with $\boldsymbol{x}^{(p)}=\boldsymbol{x}$ until $c_{x}{ }^{(p)}=0$ recursively. Equations (4)-(6) are element-wise computation for $1 \leq i \leq n$. Note that Equation (2) becomes $c_{x}(p):=0$ when $\max _{1 \leq i \leq n}\left|x^{(p)}{ }_{i}\right|=0$.

$$
\begin{aligned}
\rho & :=\left\lceil-\log _{2} \mathrm{u}_{\mathrm{b} 128}+\frac{\log _{2} \mathrm{u}_{\mathrm{b} 64}+\log _{2} n}{2}\right\rceil \\
c_{x}(p) & :=\left\lceil\operatorname{ll}_{\mathrm{b} 128}\left(\log _{2}\left(\max _{1 \leq i \leq n}\left|\boldsymbol{x}^{(p)} i\right|\right)\right)\right) \\
\sigma & :=0.75 \cdot 2^{\rho+c_{x}(p)} \\
\underline{\boldsymbol{x}}^{\prime} & :=f l_{\mathrm{b} 128}\left(\left(\boldsymbol{x}_{i}^{(p)}+\sigma\right)-\sigma\right) \\
\boldsymbol{x}^{(p+1)} & :=f l_{\mathrm{b} 128}\left(\boldsymbol{x}_{i}^{(p)}-\underline{\boldsymbol{x}}^{\prime}{ }_{i}\right) \\
\underline{\boldsymbol{x}}^{(p)}{ }_{i} & :=f l_{\mathrm{b} 128}\left(2^{-c_{x}(p)} \underline{\boldsymbol{x}}^{\prime}{ }_{i}\right)
\end{aligned}
$$

In Equation (3), the constant 0.75 was introduced by [23]. Then, $\boldsymbol{x}$ is transformed as follows.

$$
\boldsymbol{x}=\sum_{p=1}^{s_{x}} 2^{c_{x}(p)} \underline{\boldsymbol{x}}^{(p)}
$$

Here, the exponential part is scaled, and $c_{x}{ }^{(p)}$ and $\underline{\boldsymbol{x}}^{(p)}$ correspond to the exponent and significand of $\boldsymbol{x}$, respectively, and can be held on 2-byte integer and binary64, respectively.

In addition to $\boldsymbol{x}$, we split $\boldsymbol{y}$ into $c_{y}{ }^{(q)}$ and $\underline{\boldsymbol{y}}^{(q)}$. As a result, $\boldsymbol{x}^{T} \boldsymbol{y}$ is transformed as follows.

$$
\boldsymbol{x}^{T} \boldsymbol{y}=\sum_{p=1}^{s_{x}} \sum_{q=1}^{s_{y}} 2^{c_{x}(p)+c_{y}(q)} \underline{\boldsymbol{x}}^{(p)^{T}} \underline{\boldsymbol{y}}^{(q)}
$$

Here, the inner products on the right-hand side can be computed using binary64 without rounding errors, which implies that the following equation is satisfied.

$$
\underline{\boldsymbol{x}}^{(p)^{T}} \underline{\boldsymbol{y}}^{(q)}=\mathrm{fl} \mathrm{l}_{\mathrm{b} 64}\left(\underline{\boldsymbol{x}}^{(p)^{T}} \underline{\boldsymbol{y}}^{(q)}\right)
$$

When this scheme is extended to matrix multiplication, the inner products on the right-hand side in Equation (8) becomes DGEMMs, and the cost of those DGEMMs becomes dominant in the entire scheme. Using vendor-provided optimized DGEMMs is a main advantage of this scheme in terms of performance and ease of implementation. However, those DGEMMs must be performed by an algorithm that follows the same or better error-bound as the naive matrix multiplication algorithm with triple loops. Here, using a divide-and-conquer approach, e.g., Strassen's algorithm, is not permitted.

If the summation in Equation (8) is computed accurately, an accurate computation result with infinite precision can be obtained. However, if the goal is to obtain binary128-level accuracy, the summation can be computed using binary 128 arithmetic. Even in that case, as rounding errors are only caused at summation, accuracy loss does not occur in terms of the inner product-wise dimension, unlike the naive matrix-multiplication algorithm. Thus, it achieves higher accuracy than the naive algorithm with binary128 arithmetic.

The performance of this scheme depends on the number of split vectors, which depends on the following three factors.

i. The inner product dimension ( $n$ in Equation (1)). 


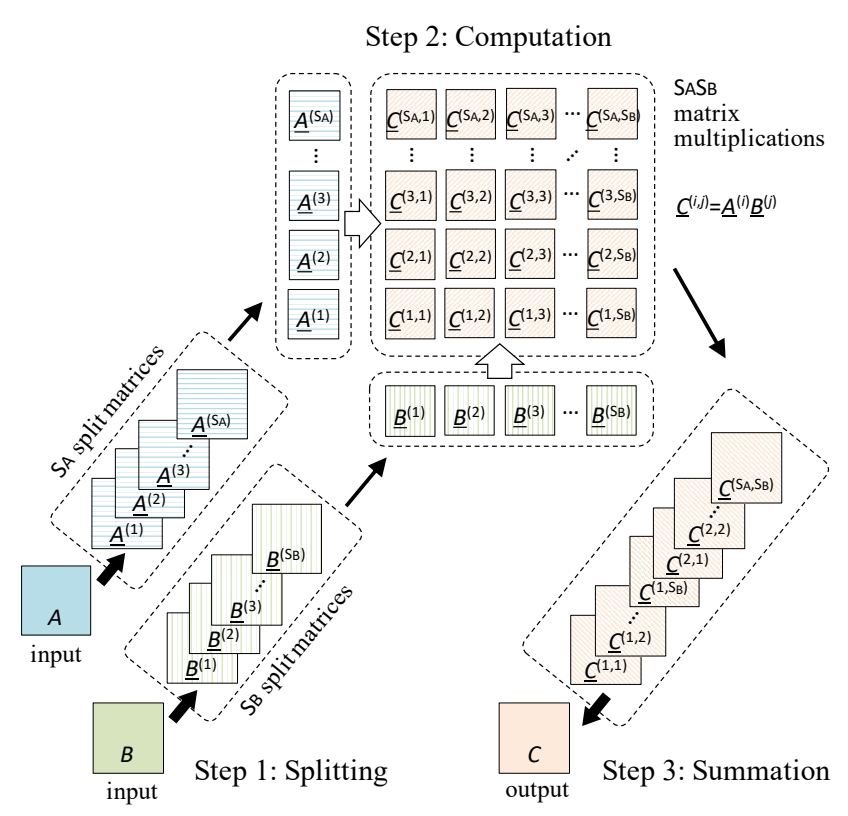

Figure 1: The Ozaki scheme on matrix multiplication $C=A B$ (scaling the exponential part is not considered).

ii. The absolute value range of the input vector elements (corresponding to max in Equation (2)).

iii. The maximum number of significant bits in the input vector elements.

In addition, memory consumption also increases with the number of split vectors.

Figure 1 shows the schematic of the Ozaki scheme on matrix multiplication $C=A B$ (scaling of the exponential part is not considered). For matrix multiplication, $n$ in Equations (1) and (2) becomes the inner product direction of the matrix multiplication (the $k$ dimension where matrix $\boldsymbol{A}$ is $m \times k$ and matrix $\boldsymbol{B}$ is $k \times n)$.

This scheme can achieve numerical reproducibility of the computed result independent of the degree of parallelism (e.g., examples of using the Ozaki scheme for reproducibility can be found in the literature [24][25]). This means that the method always returns unique results, whereas floating-point operations are nonassociative, and the results can change at the level of rounding errors when executed in environments with different degrees of parallelism. Reproducibility is achieved naturally because the scheme performs infinite precision operations, except for the summation part, where the order of the summation can be fixed (as it is elementwise). However, the precision of the log used in Equations (1) and (2) is not defined by the IEEE standard and may vary depending on the binary128 implementation and environment, and this reproducibility must be guaranteed using some approximate calculation or tables. However, this is not a problem when using the same language environment and architecture.
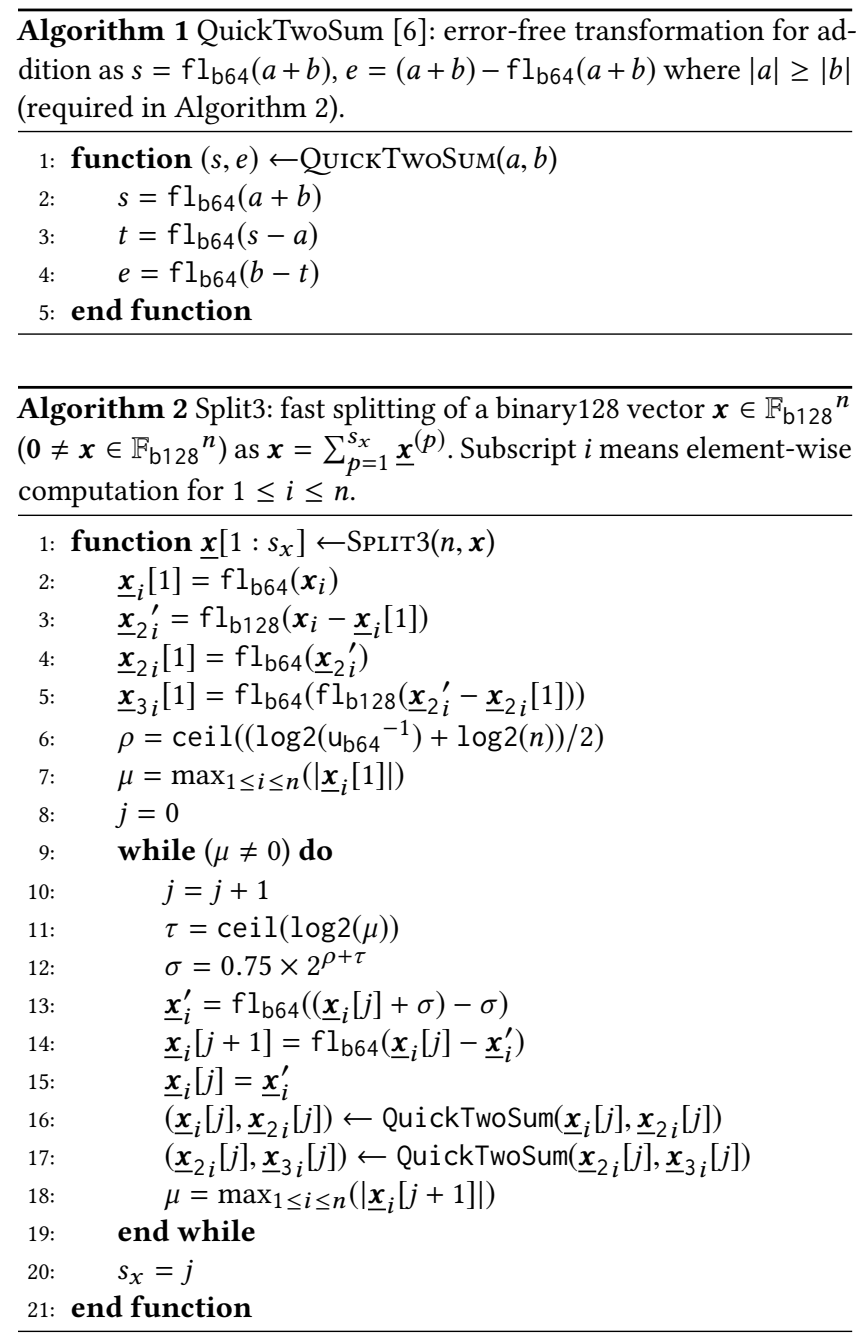

\section{OPTIMIZATIONS}

The previous section presented a minimal way to compute the matrix multiplication on binary128 using the Ozaki scheme. Here, we present several optimization methods to increase computation speed, improve accuracy, and reduce memory costs. This implementation applies all optimizations except those discussed in Section 4.4 .

\subsection{Reduction of binary 128 operations}

When computing the matrix multiplication on binary 128 using the Ozaki scheme, binary128 operations are required in the splitting and summation processes (Section 3). Although the most computationally heavy component of the Ozaki scheme is the matrix multiplications (using DGEMM), the splitting and summation processes can become a performance bottleneck because binary128 emulation is slow. To address this issue, we reduce the number of binary 128 operations in the splitting and summation processes. However, these methods use binary64 operations rather than binary128 operations; thus, they can only be used when the input is in the exponential range of binary64. 

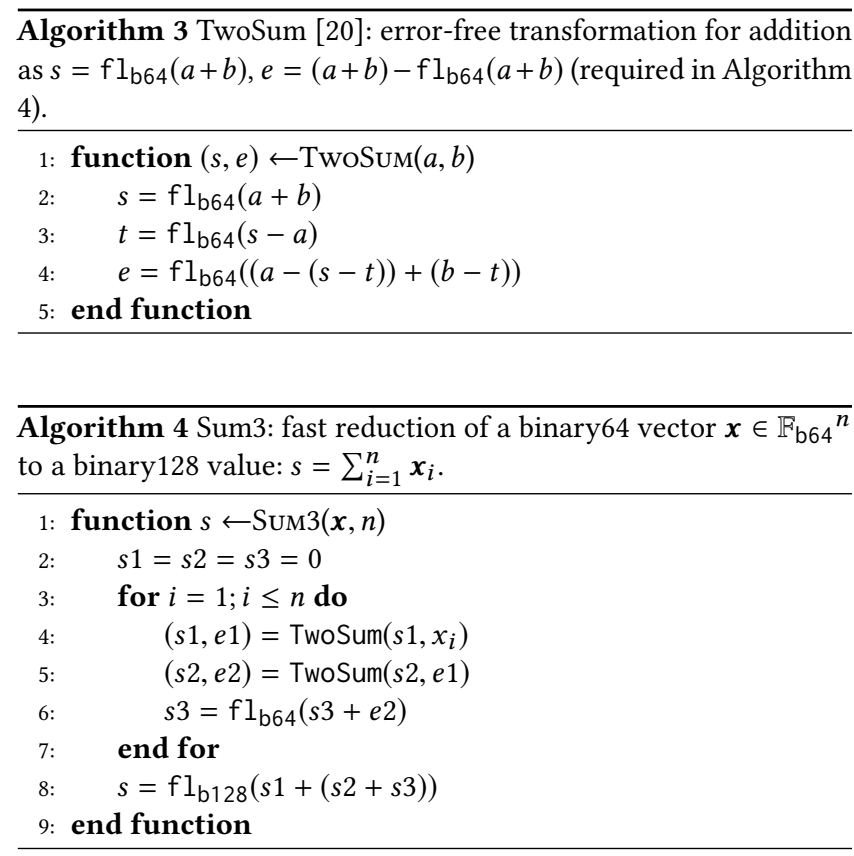

4.1.1 Fast splitting (Split3). Algorithm 2 "Split3" (with Algorithm 1) splits a binary128 vector to binary64 vectors using three binary64 bins. Here, the binary128 arithmetic is required only at lines 3 and 5. This algorithm corresponds to the processes performed in Equation (7) with Equations (1)-(6) in Section 3 with the following modifications.

- Lines 2-5: an input binary128 vector $x$ is uniquely split into three binary64 vectors such that $x=\underline{x}+\underline{x}_{2}+\underline{x}_{3}$ with $\left|\underline{x}_{i}\right| \geq$ $\left|\underline{x}_{2 i}\right| \geq\left|\underline{x}_{3 i}\right|$ (113 bits are held from the top as 53 bits +53 bits +7 bits).

- Line 6: $\mathrm{u}_{\mathrm{b} 128}$ is replaced by $\mathrm{u}_{\mathrm{b} 64}$ in Equation (1) because lines 11-14 perform the splitting with the binary64 vector $\underline{x}$ as input. Thus, we can use the splitting algorithm in the original Ozaki scheme [34].

- Line 15: $2^{-c_{x}^{(p)}}$ is not multiplied to $\underline{x}^{\prime}$ in Equation (6) because the input is binary64 (no scaling is required). Accordingly, in Equation (7), $2^{c_{x}^{(p)}}$ is eliminated.

- Lines 16-17 are added to handle the three binary64 input vectors.

4.1.2 Fast summation (Sum3). Algorithm 4 (with Algorithm 3) performs the reduction of a binary 64 vector to a binary 128 value with 159-bit precision. This algorithm is an adaptation of VecSum [35][33]. Note that $n$ in this algorithm becomes $s_{x} s_{y}$ in Equation (8). This algorithm computes the summation using three binary64 accumulators with an error-free transformation TwoSum (Algorithm 3) and uses the binary 128 arithmetic only at line 8 . This method may result in an overflow of the sum result even if the input is within the range of binary64; thus, the result must be checked. Note that it is possible to increase computation speed using two accumulators rather than three; however, the precision becomes 106 bits, which is a somewhat worse than the 113 bits of binary 128 .

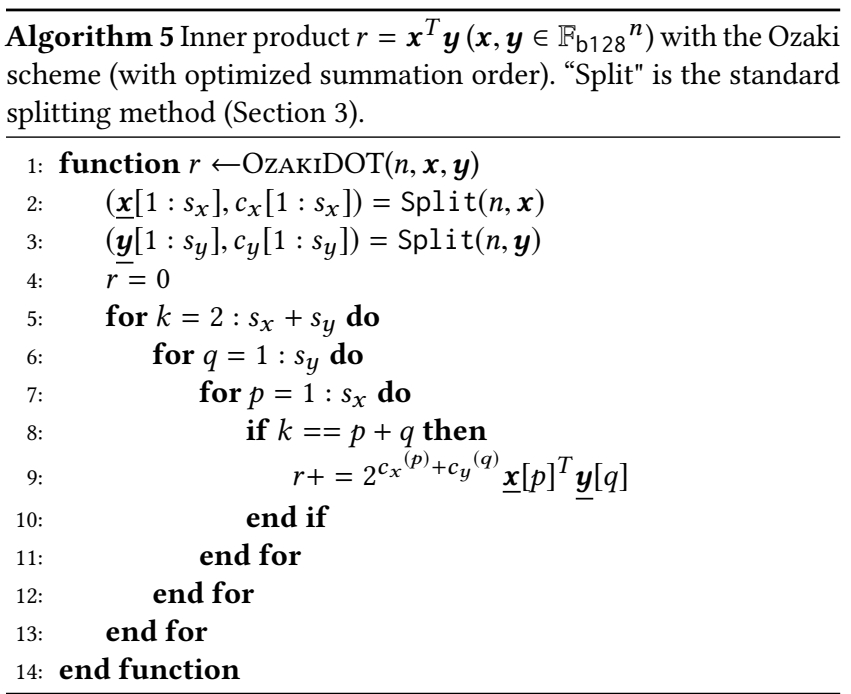

\subsection{Optimizing the summation order}

In Equation (8), the order of the two sums is arbitrary; however, the order can be devised to improve accuracy in ill-conditioned cases. Here, the smaller $p$ and $q$ are, the digits with larger absolute values are held in $\underline{\boldsymbol{x}}^{(p)}$ and $\underline{\boldsymbol{y}}^{(q)}$, respectively. Therefore, accumulating the data in ascending order of $p+q$ will improve accuracy under good conditions. However, a large accuracy loss may occur when a large cancellation occurs during the accumulation. Therefore, accumulating the data in decreasing order of $p+q$ is suggested to avoid this problem. Algorithm 5 shows the inner product using the Ozaki scheme with the optimized summation order (note this algorithm does not adopt Split3 and Sum3).

\subsection{Inner product-wise blocking}

When computing matrices, the proposed implementation consumes huge memory spaces to store split matrices, which can restrict the problem size that can be computed. This problem can be solved easily by performing the entire procedure (i.e., splitting, computation, and summation) in a block manner by dividing a matrix into a rectangle along with the inner product direction, as implemented in the literature [24][26]. However, if the block size is too small, the execution efficiency of the GEMMs used in the computation may decrease.

\subsection{Other optimization possibilities}

As mentioned in the literature [26], the following optimizations can be applied to compute matrices but are not used in the proposed implementation for simplicity.

4.4.1 Batched computation of GEMM. Multiple matrix multiplications of split matrices can be computed independently and concurrently. When the number of cores is large and the matrix size is small, using batched GEMM [8] can improve performance. The effectiveness has been demonstrated in the literature [24]. However, with this technique, space is required to store the results of all the matrix multiplications. 
4.4.2 Sparse computation. Split matrices may contain many zero elements when the range of values in the input matrix is relatively wide. Performance can be improved with a sparse matrix representation when fast sparse matrix-matrix multiplication is available [34]. The effectiveness of this approach has been demonstrated in the literature [16].

4.4.3 Outer-product-wise blocking. Since the inner product dimension ( $n$ in Equation (1)) is one factor that determines the number of split matrices (and the number of matrix multiplications), we may be able to reduce the number of split matrices by performing the Ozaki scheme in a block manner such that the inner product dimension becomes small. However, this will increase the number of summations.

4.4.4 Fast-mode with determination of the optimal number of split matrices. The Ozaki scheme performs $d^{2}$ matrix multiplications, where the number of split matrices is $d$. Here, the infinite precision operation is performed. In the literature [26], two methods have been used to reduce the number of matrix multiplications. The first method estimates the minimum $d$ required to achieve an equivalent accuracy to that obtained by the naive matrix multiplication algorithm with triple-loop, and the second method (fast-mode) omits some of the calculations for the lower bits (it reduces the number of matrix multiplications to $d(d+1) / 2$ from $\left.d^{2}\right)$. However, the former method to estimate the minimal $d$ requires binary 128 matrix-vector multiplication $d$ times, which incurs a large overhead with binary 128 emulation. The latter method can often achieve accuracy that is similar to naive matrix multiplication but may reduce accuracy in some cases depending on the input matrices condition. Thus, both methods must be used together.

\section{EVALUATION}

\subsection{Implementation}

The proposed implementation to compute the matrix multiplication $C=A B$ is coded in $C++$ and OpenMP on $x 86$. The splitting and summation processes are parallelized using OpenMP by applying the parallel for directive to the outermost loop where memory accesses are discontinuous. The computation part uses the DGEMM routine in Intel Math Kernel Library (MKL) ${ }^{14}$. To optimize the memory access in the splitting of matrix $A$, the matrix is transposed by mkl_zomatcopy in advance (there is no matcopy routine for binary128; thus, it is executed by passing a pointer to a routine for binary64 complex). Accordingly, DGEMM calls the TN-kernel (matrix $\boldsymbol{A}$ is transposed). For the inner product-wise blocking (Section 4.3), our implementation automatically selects the largest block size that can be computed within a pre-allocated work memory size. The work memory allocation is performed by an external initialization routine; thus, it is not included in the routine execution time.

\subsection{Experimental setup}

We conducted evaluations using Intel Xeon Gold 6126 processor (Skylake, 2.6-3.7 GHz, 12 cores) $\times$ two sockets with 192 GB DDR42666 RAM (255.9 GB/s), and Linux kernel 3.10.0-1160.15.2.el7.x86_64

\footnotetext{
${ }^{14}$ https://software.intel.com/oneapi/onemkl
}

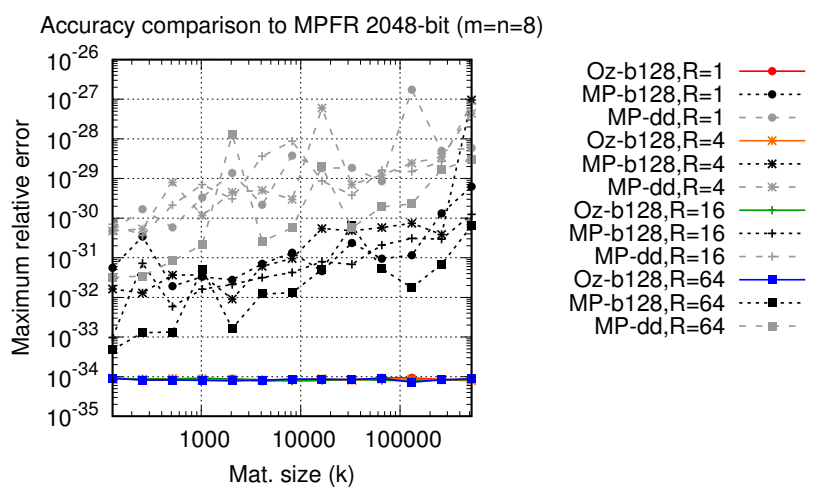

Figure 2: Maximum relative error of $\mathrm{Oz}-\mathrm{b} 128, \mathrm{MP}-\mathrm{b} 128$, and MP-dd on different input ranges (varied by $R$ ), compared to the 2048-bit MPFR operation. All the Oz-b128 results overlap.

on the Cygnus supercomputer at the University of Tsukuba. This CPU (one CPU) has 998.4 GFlops of the theoretical peak performance on binary64 at $2.6 \mathrm{GHz}$ with AVX-512. The programs were executed with one thread/core ( 24 threads in total) and with "numactl --localalloc" to ensure that memory was always allocated from the local node in the two-socket Non-Uniform Memory Access (NUMA) architecture.

We compared the following three implementations in this evaluation.

- Oz-b128: The proposed binary128 implementation based on the Ozaki scheme with Split3 and Sum3.

- MP-b128: The RGEMM routine in MPLAPACK using binary128 emulation.

- MP-dd: The RGEMM routine in MPLAPACK using DD arithmetic in the QD library.

The MPLAPACK's implementations are based on the naive matrixmultiplication algorithm with triple loops parallelized using OpenMP. The versions of the numerical libraries used in this evaluation were the Intel MKL 19.1.3 (for DGEMM in the proposed implementation) and MPLAPACK v0.9.3 with QD version 2.3.22. All codes were compiled with $\mathrm{g}++$ 8.3.1 with $-\mathrm{O} 3$.

The number of split matrices, which determines performance, depends on the absolute range of the input; thus, we initialized the input matrices with a pseudo uniform random number $\left[1,10^{R}\right.$ ) with a random sign and measured performance at different $R$ values. For the proposed implementation, 64GB was allocated as work memory (this can be reduced by applying the inner-product-wise blocking (Section 4.3)). The averaged result of three consecutive runs of the same routine was used as the measurement result.

\subsection{Accuracy}

Figure 2 shows the maximum relative error of Oz-b128, MP-b128, and MP-dd on different input ranges (varied by $R$ ), compared to the 2048-bit MPFR operation. This comparison was performed on the MPFR 2048-bit format. The proposed implementation does not incur rounding errors (i.e., infinite-precision operation) except for 

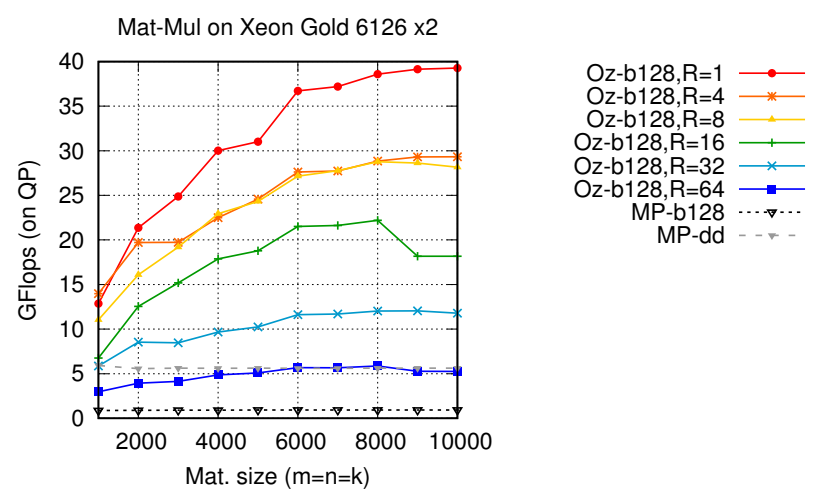

Figure 3: Throughput of $\mathrm{Oz}-\mathrm{b} 128$ with different input ranges (varied by $R$ ) and MPLAPACK's RGEMM routines.

the summation; thus, there is no reduction of accuracy for the inner product dimension, unlike the naive matrix-multiplication algorithm. In addition, Sum3, which accumulates at three times the precision of binary64, was used; thus, the summation part of the Ozaki scheme was also computed with higher precision than binary128. Consequently, the proposed implementation achieved higher accuracy than MP-b128 using binary128 arithmetic.

\subsection{Throughput}

Figure 3 shows the throughput of Oz-b128 on different input ranges (varied by $R$ ), MP-b128, and MP-dd on $R=1$. "Flops (on QP)" in the $\mathrm{y}$-axis represents the value obtained by $2 n^{3} / t$, where $t$ is execution time.

Oz-b128 outperformed MP-b128 in all cases, and MP-dd was comparable to Oz-b128 when $R=64$. Note that the DD arithmetic in the QD library used in MP-dd is not SIMD-optimized, and both MPLAPACK's routines are not as optimized as vendor's DGEMM yet; thus, there is room for further speedup in the MPLAPACK routines. The throughput of Oz-b128 is determined by the number of DGEMM calls and DGEMM throughput because the total execution time is dominated by the DGEMM execution time, as shown in Figure 4. Figure 5 shows the number of split matrices and the throughput of DGEMM used in the Oz-b128 computation. Here, "Flops" is obtained as $d^{2} \times 2 n^{3} / t_{\text {DGEMMs }}$, where $d$ is the number of split matrices and $t_{\text {DGEMMs }}$ is the execution time of DGEMMs shown in Figure 4. As shown in Figure 5 (a), the number of split matrices increases depending on the absolute range of the input, which is varied by $R$, and the inner product dimension. In the following performance evaluations, we only show the performance for $R=1$; however, as in this evaluation, the performance varied with different $R$ values.

Figure 6 shows the performance of Oz-b128 without Split3 and Sum3. Compared to the case with Split3 and Sum3, at $n=10000$, the total execution time was reduced to $83 \%$ by increasing the splitting and summation costs approximately 2.7 and 2.1 times, respectively.

\section{EXTENSIONS}

This section discusses the possibilities of GPU acceleration, SGEMMbased implementation, reduced-precision performance, performance
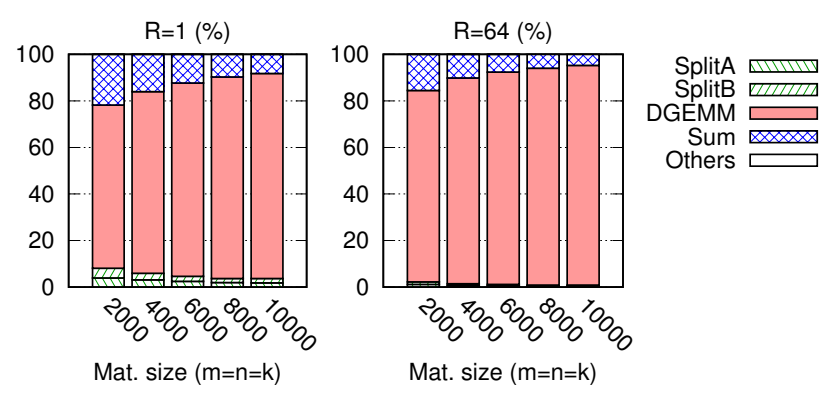

Figure 4: Execution time breakdown of Oz-b128 $(R=1,64)$. "SplitA/B" represent splitting for matrix $A / B$.

(a) Num. of Split Mat.
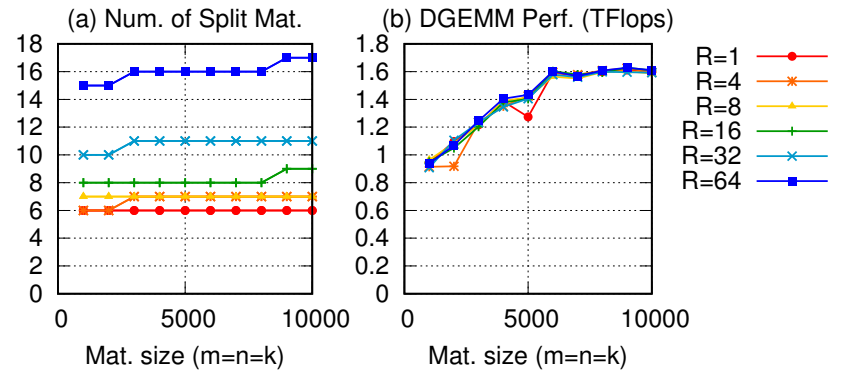

Figure 5: (a) Number of split matrices and (b) throughput of DGEMM used in the computation on Oz-b128.
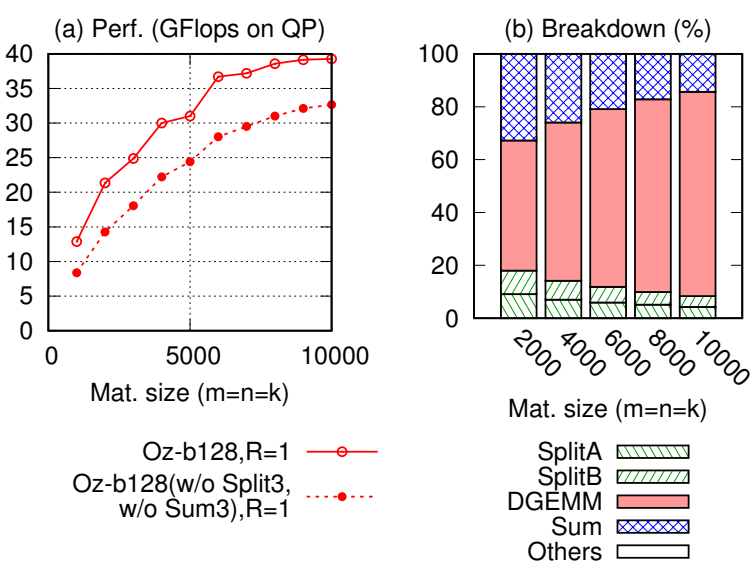

Figure 6: Performance of Oz-b128 without Split3 and Sum3 $(R=1)$ : (a) throughput and (b) execution time breakdown.

on matrix-vector multiplication, and a distributed parallel implementation. The experimental conditions used in this section are the same as those identified in the previous section. 
(a) Perf. (GFlops on QP)

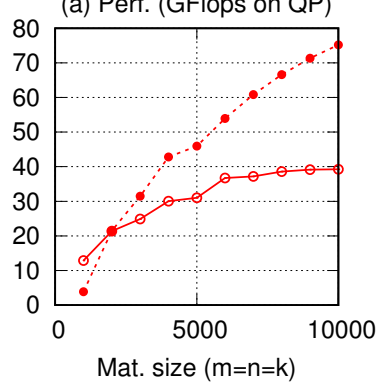

Oz-b128(+GPU), R=1 $\cdots \cdot \cdot \cdots$ $\mathrm{Oz}-\mathrm{b} 128, \mathrm{R}=1 \longrightarrow$ (b) Breakdown (\%)

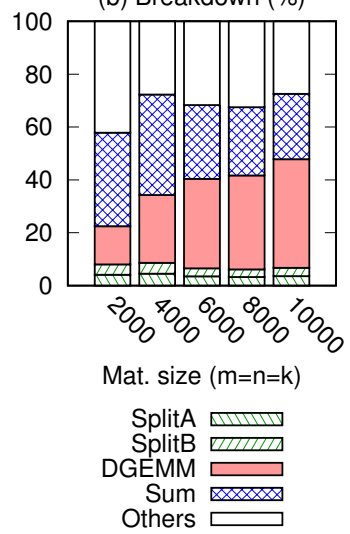

Figure 7: Performance of $\mathrm{Oz}-\mathrm{b} 128$ with and without GPU acceleration using a Tesla V100 $(R=1)$ : (a) throughput and (b) execution time breakdown.

\subsection{GPU acceleration}

The proposed implementation enables GPU acceleration by offloading DGEMM to GPUs. Note that there is no software implementation of binary128 that supports GPUs; thus, the matrix splitting and summation processes must be executed on CPUs.

The easiest way to offload DGEMM to GPUs is to use NVBLAS ${ }^{15}$ provided by NVIDIA. NVBLAS works through the CBLAS interface, and the data transfer between CPUs and GPUs is managed automatically. However, it causes unnecessary data transfer because matrices can be reused in the matrix multiplications computed in the Ozaki scheme. Therefore, we manually manage the data transfer and perform the calculations in cuBLAS. All split matrices for matrices $\mathrm{A}$ and $\mathrm{B}$ were transferred to the GPU and then computed using cublasDgemm.

We evaluated the performance using an NVIDIA Tesla V100PCIE-32GB (Volta, $1.370 \mathrm{GHz}, 80 \mathrm{SMs}, 2560$ binary64 cores), HBM2 32GB 898.0 GB/s, and CUDA 11.2.1. Here, the GPU was connected to the machine used in the previous evaluations. Figure 7 shows the throughput and execution time breakdown. As shown, the throughput was improved by GPU acceleration. This GPU has approximately 7 TFlops of theoretical peak performance on binary64, and 6.8 TFlops was observed on DGEMMs performed in this evaluation. In the execution time breakdown, "Others" includes the GPU memory allocation cost and data transfer cost between the GPU and CPU via PCIe ${ }^{16}$.

The proposed implementation calls multiple DGEMMs that can be computed independently and concurrently; thus, it is easy to take advantage of multiple GPUs. Here, rather than distributing a single GEMM across multiple GPUs, we can distribute multiple GEMMs across multiple GPUs.

\footnotetext{
${ }^{15} \mathrm{https} / /$ docs.nvidia.com/cuda/nvblas

${ }^{16}$ The host memory was not allocated using cudaMallocHost (i.e., it was not pinned); using it may improve the data transfer speed.
}

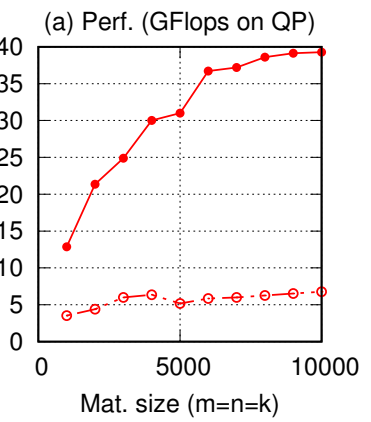

(b) Num. of Split Mat.

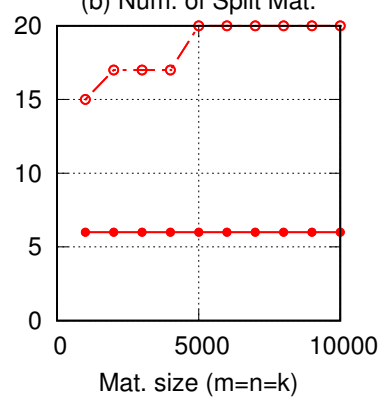

$\mathrm{Oz}-\mathrm{b} 128(\mathrm{w} / \mathrm{b} 64), \mathrm{R}=1$ $\mathrm{Oz}-\mathrm{b} 128(\mathrm{w} / \mathrm{b} 32), \mathrm{R}=1$

Figure 8: Performance of SGEMM-based Oz-b128 (Ozb128(w/b32)) and DGEMM-based Oz-b128 (Oz-b128(w/b64)) $(R=1)$ : (a) throughput and (b) the number of split matrices.

\subsection{SGEMM-based implementation}

We demonstrate the use of binary32 (SGEMM) instead of binary64 (DGEMM) for the computation part. This can be achieved by simply replacing $u_{b 64}$ with $u_{b 32}$ in Equation (1). However, if Split3 and Sum3 are used, they must be modified; i.e., to achieve binary128 precision or higher, it is necessary to expand the number of bins to five from three when using binary32. In addition, Split3 and Sum3 can only be used if the exponent range is equivalent to binary32. In the following demonstration, for simplicity, we do not use Split3 and use the binary64 implementation of Sum3. However, using SGEMM for the computation increases the number of split matrices, which makes the execution time of the computation part sufficiently large; thus, the cost of splitting and summation becomes relatively small, and the need for Split3 and Sum3 decreases.

Figure 8 shows the throughput and number of split matrices of Oz-b128 with SGEMM and that with DGEMM. In this environment, there is no advantage to using binary32 instead of binary64 for the computation. The binary32 throughput of this processor is twice that of binary64 (the observed SGEMM throughput in the evaluation was approximately 3.1 TFlops on binary32); however, the increase in computational complexity, which depends on the number of split matrices, when using binary32 is much greater than that. Nevertheless, in environments where binary64 performance is limited, as in some GPU products, the use of binary32 may contribute to performance improvement. In addition, as shown in the literature [26], the split matrices prepared for computation by SGEMM fall within the binary16 representation range; thus, they can be computed using cublasGemmEx with FP32 precision Tensor Cores operations on NVIDIA GPUs.

\subsection{Reduced precision performance}

The number of split matrices depends on the number of significand bits on the input matrices, the inner product dimension, and the absolute range of the input matrices; thus, if the input data are, for example, triple precision, throughput will be better than that of quadruple precision data. 
(a) Perf. (GFlops on QP)

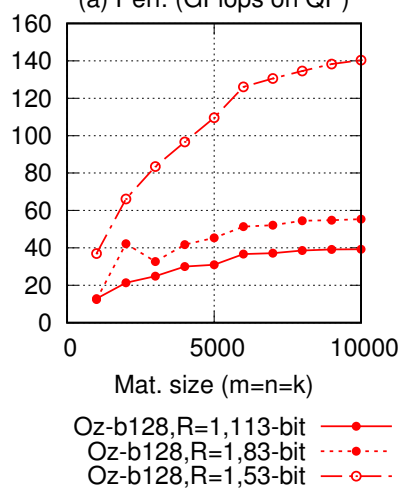

Figure 9: Performance of $\mathrm{Oz}-\mathrm{b} 128$ with reduced-precision inputs $(R=1)$. (a) throughput and (b) the number of split matrices.

Figure 9 shows the performance obtained with inputs with an 83-bit significand (equivalent to triple precision) and 53-bit significand (equivalent to binary64). This evaluation was conducted by filling a part of the lower bits of the binary128 format data with zeros, and the result was obtained with full binary128 precision. Note that the computational complexity of the proposed implementation increases with the square of the number of split matrices. Accordingly, the triple precision performance is not exactly halfway between double and quadruple precision.

\subsection{Matrix-vector multiplication}

The Ozaki scheme can also be used for other linear algebra kernels comprising inner products. Here, we demonstrate the performance of matrix-vector multiplication using the proposed implementation. In the implementation, multiple split vectors (for vector $\boldsymbol{x}$ ) were packed and treated as a single dense matrix, as was performed in the literature [24]; thus, the computation can be performed by DGEMM instead of the binary64 matrix-vector multiplication routine (DGEMV).

Figure 10 shows the throughput of matrix-vector multiplication for $\boldsymbol{y}=\boldsymbol{A x}$. Here, "Flops (on QP)" in the y-axis represents the value obtained by $2 n^{2} / t$, where $t$ is execution time. The proposed implementation (Oz-b128) outperformed MPLAPACK's RGEMV routine using binary128 emulation (MP-b128) but was slightly slower than RGEMV using the DD arithmetic (MP-dd) even on $R=1$. Compared to GEMM, GEMV is more memory intensive; thus, the memory accesses can compensate for the low performance of the binary 128 emulation and DD arithmetic to some extent. Figure 11 shows the execution time breakdown. As can be seen, the matrix splitting cost dominated the execution time. Note that this cost is proportional to the number of split matrices.

\subsection{Distributed parallel implementation}

Although we cannot report results at this time, we briefly discuss the possibility of a distributed parallel implementation. For matrix multiplication on multiple nodes, there are two options for implementation.
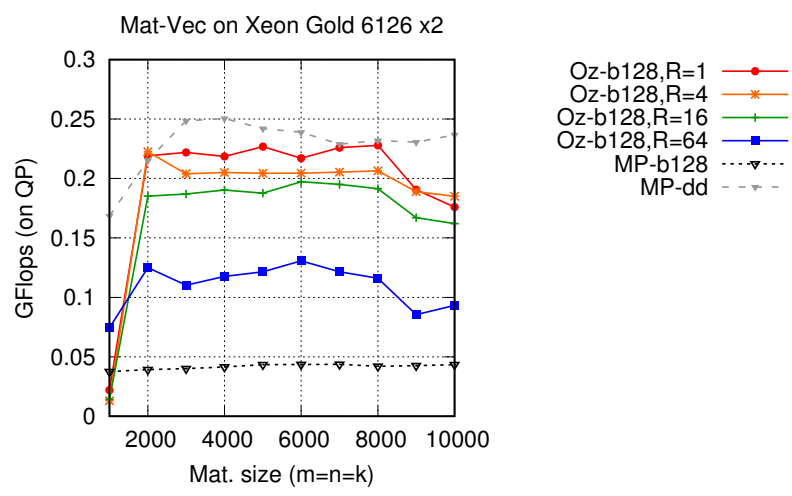

Figure 10: Throughput of matrix-vector multiplication using $\mathrm{Oz}-\mathrm{b} 128$ with different input ranges (varied by $R$ ) and MPLAPACK's RGEMV routines.
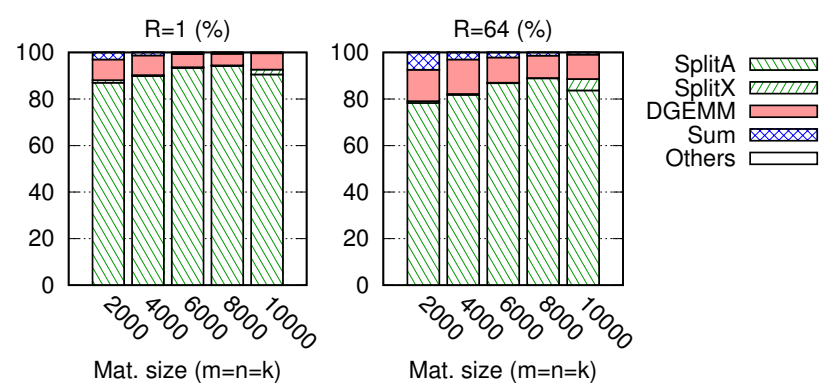

Figure 11: Execution time breakdown of matrix-vector multiplication using $\mathrm{Oz}-\mathbf{b} 128(R=1,64)$. "SplitA/X" represents splitting for matrix $A$ and vector $x$.

(1) We can parallelize the entire Ozaki scheme using the PDGEMM routine in Parallel Basic Linear Algebra Subprograms (PBLAS) [5]. The advantage of this method is that reproducibility and infinite precision operations can be achieved because it preserves the nature of the Ozaki scheme. However, this method would require a distributed parallel implementation of the matrix splitting algorithm in the Ozaki scheme. In addition, performance tends to be communication bound compared to the latter method when the problem size per node is small.

(2) We can construct a distributed parallel matrix multiplication using a classic algorithm (e.g., CANNON[4] and SUMMA[37]) in the binary 128 format. Here, the binary 128 GEMM to be executed at each node would be computed using the proposed implementation using the Ozaki scheme. This approach would eliminate the disadvantages of the former method. However, reproducibility and infinite precision operation could not be achieved because the nature of the Ozaki scheme is only preserved on the GEMM performed on each node.

\section{SUMMARY}

In this paper, we have presented a fast implementation of accurate matrix multiplication in the binary 128 format using the Ozaki 
scheme, and we have demonstrated the performance of this implementation on $\mathrm{x} 86$ processors. The advantages and disadvantages of the proposed implementation are summarized as follows.

Advantages:

- High-performance and low development cost: With the proposed implementation, it is easy to obtain higher performance than existing quadruple-precision matrix multiplication implementations using binary128 emulation and DD arithmetic on many-core processors because most computations can be performed using DGEMM, whose highly optimized implementation is available in vendor BLAS, which eliminates the performance optimization costs (including SIMD vectorization).

- Acceleration: the proposed implementation can be accelerated easily using accelerators that provide DGEMM, e.g., GPUs.

- Accurate: the proposed implementation can achieve higher accuracy than triple-loop naive matrix multiplication algorithm with binary128 or DD arithmetic.

- Reproducible: the proposed implementation can always return a bit-wise identical result for the same input even with different degrees of parallelism.

Disadvantages:

- The throughput of the proposed implementation depends on the input matrices, i.e., the wider the absolute value range of the elements of the input matrices, the worse the performance.

- High memory consumption. Similarly, more memory consumed as the width of the absolute value range of the elements of the input matrices increases. However, this issue can be relaxed somewhat by blocking techniques.

In terms of the throughput, it is difficult to make a simple comparison with existing methods/implementations because the throughput of the proposed implementation depends on the conditions of the input matrices, and the implementations of the other methods evaluated in our experiment could possively be optimized further (e.g., DD arithmetic can be SIMD optimized). However, the proposed implementation can easily achieve high performance because most computations can be performed on vendor-optimized DGEMM, e.g., on many-core processors with SIMD architectures.

In addition, the proposed implementation is extendable because it can be used for quadruple and other precisions (and different precisions of the input data and arithmetic). We expect that this work will contribute to realizing high-precision and accurate matrix multiplication (and other linear algebraic operations) on low-precision floating-point units (FPUs) and help in determining the precisions of FPUs that the hardware should support and its ratio (which is often binary32: binary64=2:1).

In future, we plan to demonstrate the practical performance benefit in applications that already use high-precision arithmetic because the performance of the proposed implementation depends on the characteristics of the input data. We also plan to compare the performance of the proposed implementation to that of FPGA implementations, including energy efficiency evaluations.

Note that the proposed implementation is available as part of OzBLAS $^{17}$.

\footnotetext{
${ }^{17}$ https://www.r-ccs.riken.jp/labs/lpnctrt/projects/ozblas/
}

\section{ACKNOWLEDGMENTS}

This research was supported by the Japan Society for the Promotion of Science (JSPS) KAKENHI Grant \#19K20286. This research used computational resources of the Cygnus supercomputer provided by Multidisciplinary Cooperative Research Program in Center for Computational Sciences, University of Tsukuba.

\section{REFERENCES}

[1] E. Anderson, Z. Bai, C. Bischof, S. Blackford, J. Demmel, J. Dongarra, J. Du Croz, A. Greenbaum, S. Hammarling, A. McKenney, and D. Sorensen. 1999. LAPACK Users' Guide (third ed.). Society for Industrial and Applied Mathematics.

[2] D.H. Bailey, R. Barrio, and J.M. Borwein. 2012. High-precision computation: Mathematical physics and dynamics. Appl. Math. Comput. 218, 20 (2012), 1010610121. https://doi.org/10.1016/j.amc.2012.03.087

[3] N. Burgess, C. Goodyer, D. R. Lutz, and C. N. Hinds. 2019. High-Precision Anchored Accumulators for Reproducible Floating-Point Summation. IEEE Trans. Comput. 68, 7 (2019), 967-978. https://doi.org/10.1109/TC.2018.2855729

[4] L. E. Cannon. 1969. A Cellular Computer to Implement the Kalman Filter Algorithm. Ph.D. Dissertation. Montana State University.

[5] J. Choi, J. Dongarra, S. Ostrouchov, A. Petitet, D. Walker, and R. C. Whaley. 1996. A proposal for a set of parallel basic linear algebra subprograms. In International Workshop on Applied Parallel Computing (PARA 1995), Lecture Notes in Computer Science, Vol. 1041. 107-114. https://doi.org/10.1007/3-540-60902-4_13

[6] T. J. Dekker. 1971. A Floating-Point Technique for Extending the Available Precision. Numer. Math. 18 (1971), 224-242. https://doi.org/10.1007/BF01397083

[7] J. Demmel, P. Ahrens, and H. D. Nguyen. 2016. Efficient Reproducible Floating Point Summation and BLAS. Technical Report UCB/EECS-2016-121. EECS Department, University of California, Berkeley.

[8] J. Dongarra, S. Hammarling, N. J. Higham, S. D. Relton, P. Valero-Lara, and M. Zounon. 2017. The Design and Performance of Batched BLAS on Modern HighPerformance Computing Systems. In International Conference on Computational Science (ICCS 2017), Vol. 108. 495-504. https://doi.org/10.1016/j.procs.2017.05.138

[9] J. J. Dongarra, C. J. Du, S. Hammarling, and I. S. Duff. 1990. A Set of Level 3 Basic Linear Algebra Subprograms. ACM Trans. Math. Software 16, 1 (March 1990), $1-17$.

[10] L. Fousse, G. Hanrot, V. Lefèvre, P. Pélissier, and P. Zimmermann. 2007. MPFR: A Multiple-precision Binary Floating-point Library with Correct Rounding. ACM Trans. Math. Software 33, 2 (2007), 13:1-13:15. https://doi.org/10.1145/1236463. 1236468

[11] J. Hauser. 2018. Berkeley SoftFloat. http://www.jhauser.us/arithmetic/SoftFloat. html.

[12] Y. Hida, X.S. Li, and D.H. Bailey. 2000. Quad-Double Arithmetic: Algorithms, Implementation, and Application. Technical Report Technical Report LBNL-46996. Lawrence Berkeley National Laboratory.

[13] Y. Hida, X. S. Li, and D. H. Bailey. 2007. Library for Double-Double and Quad-Double Arithmetic. Technical Report. NERSC Division, Lawrence Berkeley National Laboratory.

[14] T. Hishinuma and M. Nakata. 2019. pzqd: PEZY-SC2 Acceleration of DoubleDouble Precision Arithmetic Library for High-Precision BLAS. In International Conference on Computational \& Experimental Engineering and Sciences (ICCES 2019), Mechanisms and Machine Science, Vol. 75. 717-736. https://doi.org/10.1007/ 978-3-030-27053-7_61

[15] R. Iakymchuk, S. Collange, D. Defour, and S. Graillat. 2015. ExBLAS: Reproducible and Accurate BLAS Library. In Numerical Reproducibility at Exascale (NRE2015) at SC'15.

[16] S. Ichimura, T. Katagiri, K. Ozaki, T. Ogita, and T. Nagai. 2018. Threaded Accurate Matrix-Matrix Multiplications with Sparse Matrix-Vector Multiplications. In 32nd IEEE International Parallel and Distributed Processing Symposium Workshops (IPDPSW 2018). 1093-1102. https://doi.org/10.1109/IPDPSW.2018.00168

[17] IEEE Computer Society. 2008. IEEE Standard for Floating-Point Arithmetic. IEEE Std 754-2008 (2008), 1-58.

[18] K. Isupov. 2020. Using Floating-Point Intervals for Non-Modular Computations in Residue Number System. IEEE Access 8 (2020), 58603-58619. https://doi.org/ 10.1109/ACCESS.2020.2982365

[19] M. Joldes, J.-M. Muller, V. Popescu, and W. Tucker. 2016. CAMPARY: Cuda Multiple Precision Arithmetic Library and Applications. In The 5th International Congress on Mathematical Software (ICMS 2016), Lecture Notes in Computer Science, Vol. 9725. 232-240. https://doi.org/10.1007/978-3-319-42432-3_29

[20] D. E. Knuth. 1969. The Art of Computer Programming Vol.2 Seminumerical Algorithms. Addison-Wesley.

[21] X. S. Li, J. W. Demmel, D. H. Bailey, G. Henry, Y. Hida, J. Iskandar, W. Kahan, A. Kapur, M. C. Martin, T. Tung, and D. J. Yoo. 2000. Design, Implementation and Testing of Extended and Mixed Precision BLAS. ACM Trans. Math. Software 28, 2 (2000), 152-205. https://doi.org/10.1145/567806.567808 
[22] K. Masui and M. Ogino. 2020. Research on the Convergence of Iterative Method Using Mixed Precision Calculation Solving Complex Symmetric Linear Equation. IEEE Transactions on Magnetics 56, 1 (2020), 1-4. https://doi.org/10.1109/TMAG. 2019.2951280

[23] A. Minamihata, K. Ozaki, T. Ogita, and S. Oishi. 2016. Improved extraction scheme for accurate floating-point summation. In The 35th FSST Annual Conference International Conference on Simulation Technology.

[24] D. Mukunoki, T. Ogita, and K. Ozaki. 2020. Reproducible BLAS Routines with Tunable Accuracy Using Ozaki Scheme for Many-core Architectures. In 13th International Conference on Parallel Processing and Applied Mathematics (PPAM 2019), Lecture Notes in Computer Science, Vol. 12043. 516-527. https://doi.org/10. 1007/978-3-030-43229-4 44

[25] D. Mukunoki, K. Ozaki, T. Ogita, and R. Iakymchuk. 2021. Conjugate Gradient Solvers with High Accuracy and Bit-Wise Reproducibility between CPU and GPU Using Ozaki Scheme. In The International Conference on High Performance Computing in Asia-Pacific Region (HPC Asia 2021). 100-109. https://doi.org/10. $1145 / 3432261.3432270$

[26] D. Mukunoki, K. Ozaki, T. Ogita, and T. Imamura. 2020. DGEMM using Tensor Cores, and Its Accurate and Reproducible Versions. In ISC High Performance 2020, Lecture Notes in Computer Science, Vol. 12151. 230-248. https://doi.org/10.1007/ 978-3-030-50743-5_12

[27] D. Mukunoki and D. Takahashi. 2012. Implementation and Evaluation of Triple Precision BLAS Subroutines on GPUs. In 2012 IEEE 26th International Parallel and Distributed Processing Symposium Workshops PhD Forum (IPDPSW 2012). 1378-1386. https://doi.org/10.1109/IPDPSW.2012.175

[28] N. Nakasato. 2011. A Fast GEMM Implementation on the Cypress GPU. ACM SIGMETRICS Performance Evaluation Review 38, 4 (March 2011), 50-55. https: //doi.org/10.1145/1964218.1964227

[29] N. Nakasato, H. Daisaka, T. Fukushige, A. Kawai, J. Makino, T. Ishikawa, and F. Yuasa. 2012. GRAPE-MPs: Implementation of an SIMD for Quadruple/Hexuple/Octuple-Precision Arithmetic Operation on a Structured ASIC and an FPGA. In 2012 IEEE 6th International Symposium on Embedded Multicore SoCs (MCSoC 2012). 75-83. https://doi.org/10.1109/MCSoC.2012.31

[30] N. Nakasato, H. Daisaka, and T. Ishikawa. 2018. High Performance High Precision Floating-Point Operations on FPGAs Using OpenCL. In 2018 International Conference on Field-Programmable Technology (FPT'18). 262-265. https: //doi.org/10.1109/FPT.2018.00049

[31] M. Nakata. [n.d.]. The MPACK; Multiple precision arithmetic BLAS (MBLAS) and LAPACK (MLAPACK). http://mplapack.sourceforge.net.

[32] M. Nakata. 2010. A numerical evaluation of highly accurate multiple-precision arithmetic version of semidefinite programming solver: SDPA-GMP, -QD and -DD.. In 2010 IEEE International Symposium on Computer-Aided Control System Design (CACSD 2010). 29-34. https://doi.org/10.1109/CACSD.2010.5612693

[33] T. Ogita, S. M. Rump, and S. Oishi. 2005. Accurate Sum and Dot Product. SIAM Journal on Scientific Computing 26 (2005), 1955-1988. Issue 6. https://doi.org/10. $1137 / 030601818$

[34] K. Ozaki, T. Ogita, S. Oishi, and S. M. Rump. 2012. Error-free transformations of matrix multiplication by using fast routines of matrix multiplication and its applications. Numerical Algorithms 59, 1 (2012), 95-118.

[35] D. M. Priest. 1991. Algorithms for arbitrary precision floating point arithmetic. In 10th IEEE Symposium on Computer Arithmetic (ARITH 1991). 132-143. https: //doi.org/10.1109/ARITH.1991.145549

[36] K. Tomonori. 2021. Acceleration of multiple precision matrix multiplication based on multi-component floating-point arithmetic using AVX2. arXiv:2101.06584 [math.NA]

[37] Robert A. van de Geijn and Jerrell Watts. 1995. SUMMA: Scalable Universal Matrix Multiplication Algorithm. Technical Report. USA. 